\title{
Role of Adalimumab Biosimilar in the Treatment of Non-Anterior Uveitis Associated with Behçet's Syndrome
}

\author{
Jurgen Sota · Stefano Gentileschi · Maria Orsetta Perfetti • \\ Bruno Frediani · Gian Marco Tosi · Luca Cantarini (D) • \\ Claudia Fabiani
}

Received: July 9, 2021 / Accepted: August 9, 2021 / Published online: August 30, 2021

(C) The Author(s) 2021

\section{ABSTRACT}

Introduction: To evaluate the efficacy of SB5, an adalimumab biosimilar, in the management of Behçet's syndrome (BS)-related uveitis.

Methods: Data from eight BS patients (16 eyes) with active non-anterior uveitis and/or systemic uncontrolled disease or performing non-medical switch to SB5 were retrospectively collected and analyzed.

Results: Complete control of uveitis was observed in all 16 eyes without relapses during follow-up. The number of relapses 12 months prior to SB5 initiation and at last follow-up was 100 and 0 per 100 patients/year, respectively $(p=0.010)$. At baseline, four eyes had active retinal vasculitis, whereas at 3 months and at last follow-up, retinal vasculitis had resolved in all of them $(p=0.018)$. Median visual acuity did not vary significantly between baseline and last

J. Sota $\cdot$ S. Gentileschi · B. Frediani · L. Cantarini $(\bowtie)$

Rheumatology Unit, Policlinico "Le Scotte", Department of Medical Sciences, Surgery and Neurosciences, Research Center of Systemic Autoinflammatory Diseases and Behçet's Disease Clinic, University of Siena, Viale Bracci 16, 53100 Siena, Italy

e-mail: cantariniluca@hotmail.com

M. O. Perfetti - G. M. Tosi - C. Fabiani Ophthalmology Unit, Department of Medicine, Surgery and Neuroscience, University of Siena, Siena, Italy follow-up visit $(p=0.109)$. No ocular complications emerged during treatment.

Conclusions: SB5 is effective in the treatment of BS-related non-anterior uveitis. It reduces uveitis relapses and controls retinal vasculitis while allowing preservation of visual acuity.

Keywords: Adalimumab; Behçet's syndrome; Biosimilar; Retinal vasculitis; Uveitis

\section{Key Summary Points}

The development of biosimilars has raised some concerns in certain aspects such as immunogenicity, antidrug antibodies formation, safety and efficacy issues.

Given the paucity of data regarding the treatment of Behçet's syndrome-related uveitis with biosimilars, we investigated the efficacy of SB5, an adalimumab biosimilar, in the management of this rare condition.

SB5 displayed encouraging results by reducing uveitis relapses and controlling retinal vasculitis while allowing preservation of visual acuity.

SB5 may constitute a valid option for effective management of Behçet's syndrome-related uveitis. 


\section{INTRODUCTION}

Uveitis along with oral and genital ulcers characterize the main clinical features of Behçet's syndrome (BS) [1]. BS-related uveitis significantly impacts patients' quality of life in several domains, beyond the sole influence on visual acuity [2]. In accordance with the European Alliance of Associations for Rheumatology (EULAR) recommendations, BS patients with posterior segment involvement should be treated with conventional immunosuppressants or biologic response modifier agents. Those with an initial or recurrent episode of acute sightthreatening uveitis should receive high-dose corticosteroids (CS) and interferon- $\alpha$ or tumor necrosis factor (TNF)- $\alpha$ monoclonal antibodies [3], with the latter being increasingly recommended as first-line corticosteroid-sparing agents. Indeed, an expert panel from the American Uveitis Society reported substantial evidence in support of the use of anti-TNF therapies for several well-recognized forms of uveitis, and strongly recommended early administration of infliximab or adalimumab (ADA) in patients with vision-threatening ocular manifestations of BS [4]. In this regard, the use of monoclonal TNF- $\alpha$ antibodies has significantly improved visual prognosis in BS patients and widened therapeutic alternatives for the management of such a sight-threatening condition. Nevertheless, the development of several ADA biosimilars after the recent patent expiration of the original product has raised some concerns in the scientific community, especially on aspects such as immunogenicity, antidrug antibody formation, and safety and efficacy issues $[5,6]$. Additionally, the demonstrated efficacy and safety profile of SB5 (Imraldi ${ }^{\circledR}$ ), an ADA biosimilar, in patients with rheumatoid arthritis may not guarantee extrapolation to other indications, since it is not a very sensitive model for the detection of potential differences between ADA biosimilars and the reference product [7]. Data regarding its efficacy in BSrelated uveitis are scarce. We herein provide our experience on BS-related uveitis treated with SB5.

\section{METHODS}

\section{Population and Study Design}

The medical charts of eight consecutive patients enrolled from December 2018 to January 2021 (16 eyes) suffering from non-anterior BS-related uveitis were retrospectively analyzed. BS diagnosis was formulated according to the International Study Group (ISG) criteria [8]. Pretreatment work-up included complete blood chemistry, liver function, hepatitis B and C serology, QuantiFERON test for active or latent tuberculosis, urine culture test and a chest X-ray exam. Cardiac and demyelinating diseases or a recent history of malignancies had been ruled out. Treatment with SB5 was started due to active uveitis and/or poorly controlled systemic disease, or because of non-medical switching. SB5 was administered subcutaneously at the standard dose of $40 \mathrm{mg}$ every 2 weeks.

Patients were evaluated by both a rheumatologist and an ophthalmologist every 3 months or in the case of necessity (relapses or problems related to safety issues).

Ophthalmological work-up included classification of uveitis according to SUN [Standardization of Uveitis Nomenclature] criteria [9], laterality of uveitis, number of uveitis relapses in the 12 months preceding SB5 initiation and during follow-up, best-corrected visual acuity (BCVA) converted to the logarithm of the minimum angle of resolution (logMAR), detection of retinal vasculitis and uveitic macular edema (UME) and/or further ocular complications occurring while on SB5 treatment. BCVA was measured with a Snellen chart in decimal fractions at any follow-up visit. The diagnosis of UME and active retinal vasculitis was based on clinical, optical coherence tomography (OCT), and fluorescein angiography (FA) findings.

\section{Aims and Endpoints}

The primary aim of the study was to evaluate the ability of the SB5 to control and/or prevent intraocular inflammation among patients with BS-related uveitis. The secondary aims addressed the following points: (1) to evaluate the 
impact of SB5 on the occurrence of retinal vasculitis, (2) to examine its influence on visual acuity, and (3) to identify new potential ocular complications during SB5 treatment.

The primary endpoint was any statistically significant difference between the number of relapses during the 12 months prior to SB5 initiation and at the time of the last follow-up visit. Relapses were defined as the occurrence of ocular inflammatory manifestations after a period of remission. The secondary aims were studied using the following endpoints: (1) resolution of retinal vasculitis assessed by FA at last follow-up visit, and (2) changes in BCVA or (3) detection of new ocular structural complications emerging during treatment with SB5.

\section{Statistical Analysis}

Data were computed using IBM SPSS Statistics for Windows, version 24 (IBM Corp., Armonk, NY, USA). Descriptive statistics were used to calculate mean or median with their respective standard deviation or interquartile range, as appropriate. The Shapiro-Wilk test was employed to assess data normality distribution. Multiple repeated categorical measures were analyzed with Cochran's $Q$ test followed by the McNemar test with Bonferroni correction for post hoc analysis. The Wilcoxon signed-rank test was used for the analysis of ordinal variables. The threshold for statistical significance was set to $p<0.05$, and all $p$ values were twosided.

This study was conducted in accordance with the Declaration of Helsinki and was approved by the Local Ethics Committee of Azienda Ospedaliera Universitaria Senese (Reference No. 14951).

\section{RESULTS}

Eight patients (six male and two female) were enrolled in the study. Mean \pm SD age at uveitis onset was $35.38 \pm 6.89$ years, mean disease duration was $14.75 \pm 7.42$ years, and mean treatment duration with SB5 was $16.38 \pm 3.93$ months. Demographic and clinical characteristics are displayed in Table 1 . With regard to the anatomical pattern, uveitis was posterior in four eyes, whereas 12 eyes had panuveitis. Retinal vasculitis was encountered in two eyes affected by posterior uveitis and in two eyes with panuveitis. One eye also had uveitic macular edema at baseline (OCT central macular thickness $487 \mu \mathrm{m})$. SB5 was administered subcutaneously at the standard dose of $40 \mathrm{mg}$ every 2 weeks. A posologic adjustment was performed in one patient (SB5 $40 \mathrm{mg}$ every 10 days) because of uncontrolled systemic disease, with remission of mucocutaneous lesions within a few weeks.

The number of ocular relapses during the 12 months prior to SB5 initiation and at the last follow-up visit was 8 (100 relapses/100 patients/ year) and 0 , respectively $(p=0.04)$. Over a mean follow-up of 16-months, complete resolution of inflammation was observed in all affected eyes. Concerning FA findings, retinal vasculitis resolved in four out of four eyes affected at baseline evaluation. A statistically significant decrease in retinal vasculitis occurrence was detected during the overall study period $(p=0.02)$. Post hoc analysis, however, did not reveal a significant difference between baseline and 3 months $(p=0.13)$ or between baseline and last follow-up visit $(p=0.13)$. Figure 1 shows the resolution of panuveitis and occlusive retinal vasculitis in a BS patient affected by bilateral ocular involvement. Median $\pm \mathrm{IQR}$ BCVA expressed in logMAR at baseline and last follow-up visit was $0.00 \pm 0.13$ and $0.00 \pm 0.05$, respectively $(p=0.11)$. No new ocular complications emerged during SB5 therapy. Treatment with SB5 was well tolerated, and no adverse events were reported.

\section{DISCUSSION}

In the present case series, we have investigated the role of SB5, an ADA biosimilar, as a potential weapon for the treatment of BS-related uveitis. TNF- $\alpha$ blockade has revolutionized the treatment of BS-related uveitis by improving visual prognosis. In this context, our working group has highlighted through real-life data the effectiveness of TNF- $\alpha$ inhibitors ADA and infliximab for the long-term management of BS- 


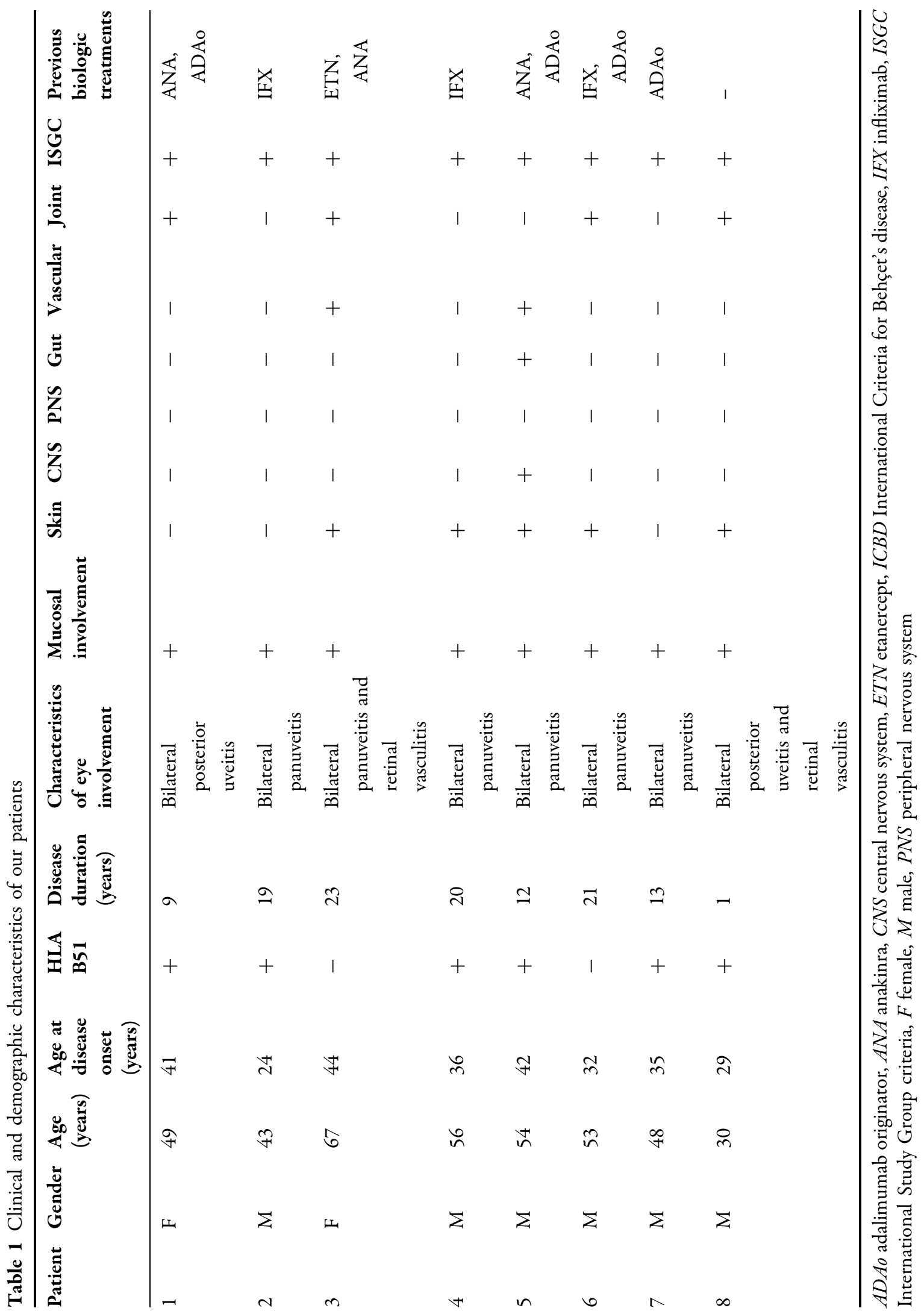



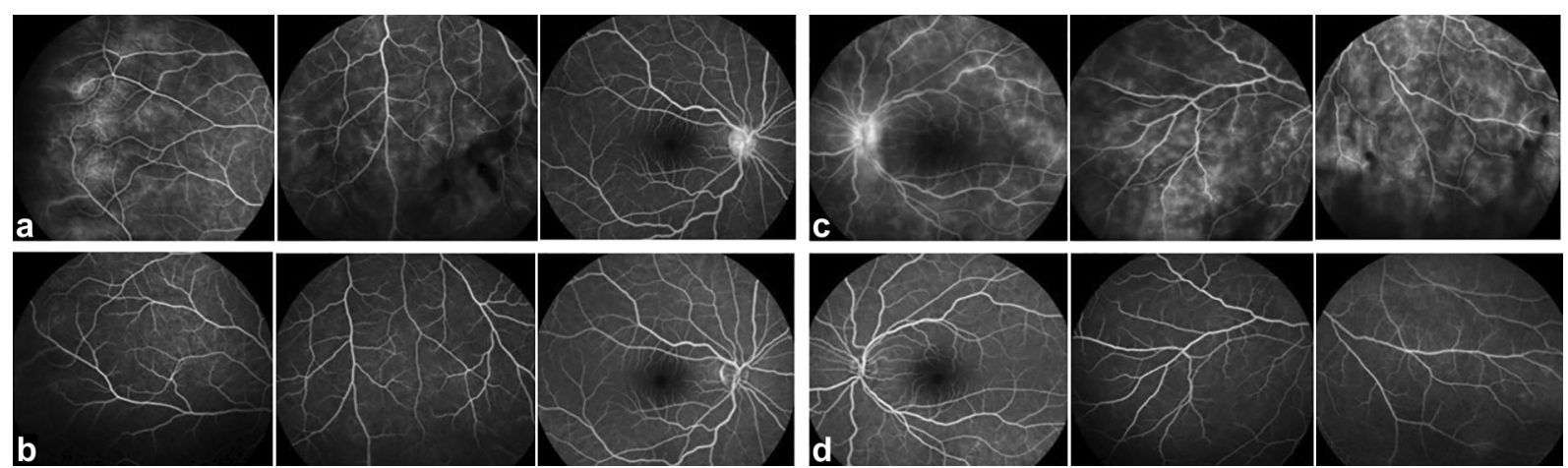

Fig. 1 Fluorescein angiography findings in a patient affected by Behçet's syndrome-related bilateral panuveitis before and after the introduction of adalimumab biosimilar SB5 monotherapy administered subcutaneously every

14 days. Late-phase angiograms show bilateral posterior pole and peripheral active retinal vasculitis characterized by optic disc and vascular leakage in the right (A) and left (C) eye, with their respective resolution in $\mathbf{B}$ and $\mathbf{D}$

related uveitis [10, 11]. High similarities between SB5 and its reference product have been reported in terms of biological characteristics and pharmacokinetic effects [12]. Nevertheless, biosimilars have raised some concerns with regard to their immunogenicity, safety profile, antidrug antibody formation and extrapolation to other indications from studies conducted in rheumatoid arthritis [5-7]. To the best of our knowledge, the present case series represents the largest sample of BS patients affected by uveitis and successfully treated with SB5. Specifically, a significant reduction in uveitis relapses was observed, which is consistent with results obtained by treating BS-related uveitis with the ADA originator. Indeed, a significant decrease in the number of uveitis relapses from 200 per 100 patients/year to 8.5 per 100 patients/year was previously reported in patients treated with the reference product [11]. Additionally, the resolution of retinal vasculitis in four out of four eyes at 3 months and its maintenance during the study period further support the rapid and sustained efficacy of SB5 in treating intraocular inflammation in BS patients. Similar findings are described in a larger cohort of patients suffering from retinal vasculitis and treated with ADA originator [13]. The availability of rapid-acting biologic agents represents a key factor in the therapy of BS-related uveitis, given the high risk of developing long-term structural damage and irreversible

visual loss, especially in patients carrying specific risk factors, such as male gender, early age at onset and uveitis duration [14]. In this context, most patients in our cohort were young men, and additionally, six out of eight were positive for HLA-B51, which may predict the development of long-term structural complications, alongside uveitis duration and the presence of panuveitis [15]. With regard to visual acuity, median BCVA did not change significantly between baseline and last followup visit. The lack of statistical differences may be explained by the high visual acuity at SB5 initiation and the early administration of biologic therapy. This may suggest the ability of SB5 to maintain visual acuity.

These findings should be interpreted with caution in the context of study limitations such as the small sample size and the lack of a prospective properly design protocol.

\section{CONCLUSIONS}

The SB5 biosimilar has been shown to be a valid option for effective management of BS-related uveitis. Indeed, SB5 efficacy was supported by its ability to dramatically reduce uveitis relapses, treat retinal vasculitis and preserve visual acuity. 


\section{ACKNOWLEDGEMENTS}

Funding. No funding or sponsorship was received for this study or publication of this article.

Authorship. All named authors meet the International Committee of Medical Journal Editors (ICMJE) criteria for authorship for this article, take responsibility for the integrity of the work as a whole, and have given their approval for this version to be published.

Authorship Contributions. Claudia Fabiani conceived the study design. Jurgen Sota performed the data analysis and wrote the first draft of the manuscript. All authors critically revised the manuscript.

Disclosures. Jurgen Sota has nothing to disclose. Stefano Gentileschi has nothing to disclose. Maria Orsetta Perfetti has nothing to disclose. Bruno Frediani has nothing to disclose. Gian Marco Tosi has nothing to disclose. Luca Cantarini has nothing to disclose. Claudia Fabiani has nothing to disclose.

Compliance with Ethics Guidelines. Written informed consent for treatment and data collection and its publication were obtained from each patient. The study was conducted in accordance with the Declaration of Helsinki and was approved by the local ethics committee of Azienda Ospedaliera Universitaria Senese (Reference No. 14951).

Data Availability. Data are available from the corresponding author upon reasonable request.

Open Access. This article is licensed under a Creative Commons Attribution-NonCommercial 4.0 International License, which permits any non-commercial use, sharing, adaptation, distribution and reproduction in any medium or format, as long as you give appropriate credit to the original author(s) and the source, provide a link to the Creative Commons licence, and indicate if changes were made. The images or other third party material in this article are included in the article's Creative Commons licence, unless indicated otherwise in a credit line to the material. If material is not included in the article's Creative Commons licence and your intended use is not permitted by statutory regulation or exceeds the permitted use, you will need to obtain permission directly from the copyright holder. To view a copy of this licence, visit http://creativecommons.org/licenses/bync/4.0/.

\section{REFERENCES}

1. Yazici H, Seyahi E, Hatemi G, Yazici Y. Behçet syndrome: a contemporary view. Nat Rev Rheumatol. 2018;14:107-19.

2. Fabiani C, Vitale A, Orlando I, et al. Quality of life impairment in Behçet's disease and relationship with disease activity: a prospective study. Intern Emerg Med. 2017;12:947-55.

3. Hatemi G, Christensen R, Bang D, et al. 2018 update of the EULAR recommendations for the management of Behçet's syndrome. Ann Rheum Dis. 2018;77:808-18.

4. Levy-Clarke G, Jabs DA, Read RW, et al. Expert panel recommendations for the use of anti-tumor necrosis factor biologic agents in patients with ocular inflammatory disorders. Ophthalmology. 2014;121:785.e3-796.e3.

5. Cline A, Feldman SR. Biologics are too complicated to duplicate: should we be worried about biosimilars? Br J Dermatol. 2018;179:557-8.

6. Rocco P, Selletti S, Minghetti P. Biosimilar switching and related medical liability. J Forensic Leg Med. 2018;55:93-4.

7. Lee H. Is extrapolation of the safety and efficacy data in one indication to another appropriate for biosimilars? AAPS J. 2014;16:22-6.

8. Criteria for diagnosis of Behçet's disease. International Study Group for Behçet's disease. Lancet. 1990;335(8697):1078-80.

9. Jabs DA, Nussenblatt RB, Rosenbaum JT, Standardization of Uveitis Nomenclature (SUN) Working Group. Standardization of uveitis nomenclature for reporting clinical data Results of the First International Workshop. Am J Ophthalmol. 2005;140: 509-16. 
10. Fabiani C, Sota J, Vitale A, et al. Cumulative retention rate of adalimumab in patients with Behçet's disease-related uveitis: a four-year follow-up study. Br J Ophthalmol. 2018;102:637-41.

11. Fabiani C, Vitale A, Emmi G, et al. Efficacy and safety of adalimumab in Behçet's disease-related uveitis: a multicenter retrospective observational study. Clin Rheumatol. 2017;36:183-9.

12. Lee JJ, Yang J, Lee C, Moon Y, Ahn S, Yang J. Demonstration of functional similarity of a biosimilar adalimumab SB5 to Humira ${ }^{\circledR}$. Biologicals. 2019;58:7-15.

13. Fabiani C, Sota J, Rigante D, et al. Efficacy of adalimumab and infliximab in recalcitrant retinal vasculitis inadequately responsive to other immunomodulatory therapies. Clin Rheumatol. 2018;37:2805-9.

14. Kural-Seyahi E, Fresko I, Seyahi N, et al. The longterm mortality and morbidity of Behçet syndrome: a 2-decade outcome survey of 387 patients followed at a dedicated center. Medicine (Baltimore). 2003;82:60-76.

15. Sota J, Cantarini L, Vitale A, et al. Long-term outcomes of Behçet's syndrome-related uveitis: a monocentric Italian experience. Mediators Inflamm. 2020;2020:6872402. 